\title{
MICROFINANCE ET MICRO-ASSURANCE SANTÉ : RÉFLEXIONS SUR DES ARTICULATIONS POSSIBLES À PARTIR DE QUELQUES EXPÉRIENCES AU BÉNIN ET AU BURKINA FASO
}

\author{
Marc Labie et al. \\ De Boeck Supérieur | Mondes en développement
}

$2007 / 3-n^{\circ} 139$

pages 57 à 71

ISSN 0302-3052

Article disponible en ligne à l'adresse:

http://www.cairn.info/revue-mondes-en-developpement-2007-3-page-57.htm

Pour citer cet article :

Labie Marc et al., « Microfinance et micro-assurance santé : réflexions sur des articulations possibles à partir de quelques expériences au Bénin et au Burkina Faso », Mondes en développement, 2007/3 n¹39, p. 57-71. DOI : 10.3917/med.139.0057

Distribution électronique Cairn.info pour De Boeck Supérieur.

(c) De Boeck Supérieur. Tous droits réservés pour tous pays.

La reproduction ou représentation de cet article, notamment par photocopie, n'est autorisée que dans les limites des conditions générales d'utilisation du site ou, le cas échéant, des conditions générales de la licence souscrite par votre établissement. Toute autre reproduction ou représentation, en tout ou partie, sous quelque forme et de quelque manière que ce soit, est interdite sauf accord préalable et écrit de l'éditeur, en dehors des cas prévus par la législation en vigueur en France. II est précisé que son stockage dans une base de données est également interdit. 


\title{
Microfinance et micro-assurance santé : réflexions sur des articulations possibles à partir de quelques expériences au Bénin et au Burkina Faso
}

\author{
Marc LABIE ${ }^{1}$, Marthe NYSSENS ${ }^{2}$ et Pascal WÉLÉ ${ }^{3}$
}

$\mathrm{D}$

ans les pays en développement, les systèmes classiques existants, notamment en matière d'intermédiation financière et de couverture sanitaire ont montré des limites se traduisant, entre autres, par l'exclusion des populations à faible pouvoir économique. En guise de relais, on assiste à l'émergence de dispositifs parallèles, tous centrés sur les micro-entrepreneurs et les ménages pauvres, à savoir la microfinance et la micro-assurance santé. La première, dont la problématique est largement développée depuis plusieurs années, groupe l'ensemble des mécanismes d'offre des services financiers adaptés aux besoins des ménages pauvres économiquement actifs n’ayant pas accès aux circuits financiers classiques. Ce faisant, elle est perçue comme un instrument pertinent de réduction de la pauvreté (Banque mondiale, 1998). Quant à la micro-assurance santé, elle suscite un intérêt grandissant dans les débats scientifiques. Face à l'implémentation, notamment en Afrique de l'Ouest, de "la politique de recouvrement des coûts" dans le domaine de la santé et son corollaire, la mise en place de systèmes payants dans l'accès aux soins primaires et secondaires, se pose la question de l'accès aux soins de santé pour toute une partie de la population. Dans ce contexte, la mise en place d'un dispositif d'assurance santé en faveur des populations démunies constitue un moyen de lutte contre la pauvreté et l'exclusion sociale (Dror et Preker, 2002).

En dépit de leurs spécificités, la microfinance et la micro-assurance sont toutes deux perçues comme étant porteuses d'impacts positifs en faveur du mieux-être

${ }^{1}$ Centre de Recherche Warocqué (UMH), Centre Emile Bernheim (SBS-ULB), Grap OSC. Le projet GRAP OSC (Groupe de Recherche en Appui à la Politique sur les Organisations de la Société Civile) est un projet soutenu par la DGCD et la CUD dans le cadre de la coopération belge. Il vise à étudier le fonctionnement et l'appui aux organisations de la société civile sous un angle pluri-disciplinaire combinant des approches économique, sociologique et anthropologique. Marc.Labie@umh.ac.be.

${ }^{2}$ UCL - IRES - Cerisis, Grap OSC.nyssens@ires.ucl.ac.be

${ }^{3}$ Université de Liège, Grap OSC. p.wele@student.ulg.ac.be 
des populations démunies. Mais bien au-delà de cette perception, certains pensent que le couplage des deux systèmes est susceptible de générer des synergies, soit en termes de renforcement de la capacité des adhérents des services de micro-assurance à faire face à leurs cotisations, soit en termes de réduction des risques de non-remboursement des crédits. Vue sous un premier angle, la micro-assurance, prise globalement, apparaît comme un instrument de gestion des risques et de renforcement des impacts des institutions de microfinance (Miller \& Northrip, 2000 ; Lai, 2000 ; Churchill, 2004 ; Brown \& Churchill, 2004). Sous un second angle, la micro-assurance santé est indispensable pour un meilleur accès aux soins de santé. Cependant, pour pouvoir payer leurs cotisations, les adhérents aux systèmes de micro-assurance peuvent avoir recours à la microfinance, dans le but d'accroître leurs capacités productives (Fonteneau \& alii, 2004).

Le présent travail vise à étudier la manière dont peuvent se combiner la microfinance et la micro-assurance santé en Afrique de l'Ouest, en vue de voir si des articulations adéquates de ces deux domaines sont susceptibles ou non de permettre une meilleure inclusion économique et sociale des populations démunies. À cet effet, nous avons réalisé une étude exploratoire de terrain en observant différents modèles institutionnels d'articulation entre microfinance et micro-assurance santé au Bénin et au Burkina Faso.

Ce document s'articule en quatre parties. La première rappelle quelques caractéristiques fondamentales de la microfinance et de la micro-assurance santé. La deuxième propose un cadre d'analyse susceptible de servir de référence pour aborder cette articulation. La troisième présente quelques exemples de couplage microfinance-micro-assurance santé au Bénin et au Burkina Faso. Enfin, la conclusion présente quelques questions qui découlent du cadre d'analyse proposé.

\section{CARACTÉRISTIQUES FONDAMENTALES DE LA MICROFINANCE ET DE LA MICRO- ASSURANCE SANTÉ}

\subsection{Le système de microfinance}

Idéalement, le bon fonctionnement d'un système de microfinance exige le respect des principes de durabilité et d'adéquation des services aux besoins et aux attentes du public cible. Le système de microfinance doit être capable de faire une offre de services qui soit meilleure que ce que proposent les marchés informels d'épargne et de crédit (soit moins cher, soit une plus grande variété de services et/ou une meilleure adaptation des produits proposés) permettant ainsi aux clients-bénéficiaires de libérer une partie des fonds précédemment utilisés pour des services financiers au profit d'autres usages plus directement utiles d'un point de vue social (nutrition, santé, éducation...). En matière d'épargne, par contre, la sécurité et la libre disponibilité des fonds jouent un 
rôle au moins aussi important que le rendement. La collecte de l'épargne devra se fonder sur le trio suivant: sécurité, disponibilité et rendement: les deux premiers éléments étant les plus essentiels (Labie, 2004, 12).

\subsection{Le système de micro-assurance santé}

En ce qui concerne les mécanismes d'assurance santé, la logique est quelque peu différente, puisqu'elle associe, comme dans toute assurance, la perception qu'a l'assuré du risque à couvrir et le coût de cette couverture. Pour que l'assuré décide de souscrire à un mécanisme d'assurance, il faut remplir trois conditions fondamentales. D'une part, il faut que l'assuré dispose de liquidités suffisantes pour pouvoir supporter le coût d'une prime jugée raisonnable et associée à un événement incertain (le fait d'être malade) sans avoir à sacrifier des revenus absolument nécessaires pour assurer des besoins qui sont eux certains (exemple de la nutrition de base). Cet élément renvoie au problème des indigents. Comment inclure dans les micro-assurances santé des personnes qui n'ont pas les moyens financiers de payer les primes d'assurance? D'autre part, il faut que l'assuré soit persuadé qu'en cas de sinistre il sera correctement couvert (sinon pourquoi choisirait-il de s'assurer?). Enfin, il faut que la personne soit convaincue que cette assurance offre des services supérieurs à ce que proposent les systèmes informels traditionnels (emprunts usuraires pour couvrir le sinistre, groupement d'entraide et de prévoyance, système de pré-paiement sans partage de risque); soit que ces services sont moins chers, soit qu'ils offrent une meilleure couverture sociale. Pour le mécanisme d'assurance, le problème est aussi simple : les fonds issus des primes collectées doivent être suffisants pour couvrir, d'une part, les sinistres qui seront déclarés dans la période suivante et, d'autre part, les coûts administratifs générés par la gestion du système.

\subsection{Les différences fondamentales}

La comparaison des deux séries de contraintes permet les remarques suivantes.

- Dans les deux cas, la gestion de la liquidité est cruciale, mais il existe une assez grande différence. Pour l'assurance santé, il est beaucoup plus difficile de prévoir les besoins de liquidités car les sinistres ne suivent évidemment pas un cycle aussi prévisible que celui associé aux renouvellement et extension de prêts (en matière d'épargne, l'argument est plus discutable).

- Dans les deux cas, la viabilité passe par une couverture de l'ensemble des coûts. Mais mis à part les coûts administratifs qui sont, dans une certaine mesure, comparables, la nature de ces coûts est très différente.

- Dans les deux cas, les bénéficiaires/clients ne participeront que si ils ont l'impression que cela améliore leur situation. Or, on peut penser qu'il existera des situations où les coûts de transaction à supporter seront tels qu'ils préféreront ne pas participer plutôt que de supporter les contraintes imposées par les systèmes institutionnels mis en place. Par ailleurs, en matière de santé, il faut que les bénéficiaires soient convaincus de la qualité acceptable des services 
auxquels ils auront accès. En effet, il faut à ce stade distinguer le produit "assurance" et le produit "offre de services de santé" que couvre l'assurance. Or, malheureusement, il semble que dans bien des cas, la qualité des soins disponibles est loin d'être uniforme. Une recherche menée sous la direction de Jaffré et Olivier de Sardan (2003) révèle des dysfonctionnements (tels que favoritisme, surfacturation, mauvaise qualité de l'accueil ou diagnostics approximatifs) qui caractérisent les systèmes de soins en Afrique de l'Ouest, à tel point que les auteurs se demandent s'il faut encore parler de dysfonctionnements ou plutôt de pratiques habituelles (Ibid., 52).

- Dans les deux cas, la proximité culturelle est fondamentale. De nombreuses recherches l'ont montré, ces mécanismes, qu'il s'agisse de microfinance ou de micro-assurance santé, ne pourront fonctionner que si ils sont adoptés par les populations concernées, ce qui suppose une méthodologie, des procédures et même un langage qui leur soient parfaitement adaptés. En cas de non-respect de cette condition, il y a fort à craindre l'exclusion des plus marginalisés. L'histoire de la microfinance est instructive à plus d'un égard à ce sujet. Qu'en est-il de l'assurance santé ? Quels sont les mécanismes traditionnels sur lesquels reposent les couvertures de sinistres ? Les micro-assurances santé peuvent-elles intégrer ces mécanismes? Il faut ici souligner deux différences fondamentales. Premièrement, le concept d'assurance santé au travers du paiement de primes est étranger aux pratiques informelles, alors que les pratiques de microfinance sont, elles, bien ancrées. Deuxièmement, dans la relation entre microcrédit et épargne et micro-assurance, le sens de la confiance est opposé. Pour le microcrédit, c'est l'organisation qui doit faire confiance au micro-entrepreneur, alors que pour l'épargne et la micro-assurance, c'est l'inverse (Churchill, 2005).

\section{CADRE D'ANALYSE}

Compte tenu de ces caractéristiques fondamentales, quelles sont les hypothèses qui peuvent être posées quant aux effets du couplage microfinance et microassurance santé ? A priori, trois types d'effets semblent susceptibles de justifier l'idée qu'une liaison entre microfinance et micro-assurance santé améliore l'inclusion sociale des populations concernées.

\subsection{Effets du couplage sur le plan du microcrédit}

On suppose qu'en associant ces deux types de services on réduit les risques de non-remboursement des clients emprunteurs pour cause de problèmes de santé (Miller \& Northrip, 2000 ; Lai, 2000 ; Churchill, 2004 ; Brown \& Churchill, 2004).

Cette hypothèse se fonde, en réalité, sur deux arguments. Le premier réside dans l'idée qu'un individu couvert par une micro-assurance santé ne devra pas "détourner" des fonds d'un usage productif pour faire face à des coûts de santé. Il s'en suivrait une diminution du risque de voir baisser la rentabilité des prêts et une augmentation de la probabilité de respect des échéances: "Il existe une 
logique de complémentarité entre les services financiers offerts par les institutions de microfinance et la micro-assurance santé. Pour un ménage à faible revenu, le microcrédit aide assurément à maintenir ou à développer des activités et l'épargne permet une stratégie de prévoyance face aux dépenses futures, programmées ou non. Cependant, une situation de crise imposant des dépenses bien au-delà de l'épargne accumulée, peut avoir un impact décisif sur ce ménage (l'obliger à se surendetter, à vendre une partie de l'outil productif) et compromettre ainsi parfois plusieurs années d'accumulation liée au crédit. Un service d'assurance permettant de faire face à ces difficultés, serait donc un outil complémentaire cohérent" (Poursat, 2004, 56).

Deuxièmement, on pense qu'un individu disposant d'une couverture médicale sera, toutes choses égales par ailleurs, en meilleure santé que son homologue n'en disposant pas, le coût du suivi en matière de santé n'étant plus un obstacle. Dans cette logique, le système micro-assurance améliore la productivité du travailleur (un travailleur en bonne santé étant a priori plus productif qu'un travailleur malade), ce qui doit logiquement déboucher sur une meilleure rentabilité de son activité, et donc sur une plus grande probabilité d'être à même de faire face à ses obligations financières.

Si d'un point de vue théorique, les deux arguments semblent pertinents, en réalité il convient d'être plus nuancé. En effet, la pratique en microfinance montre que la fongibilité des petits crédits est un fait qui est bien plus généralisé que la seule question du "détournement pour soins de santé". À titre d'exemple, des travaux ont montré que très souvent un individu se fait financer pour une extension de son activité principale et utilise le financement ainsi obtenu pour lancer une autre activité (Botzung, 1996). Si cela peut sembler anormal du point de vue de la relation entre client/bénéficiaire et institution de crédit, cela se comprend quand on sait que les micro-entrepreneurs cherchent avant tout à générer une diversification de leurs activités afin de répartir les risques encourus. Si on accepte cet état de fait, on comprend que lutter contre la fongibilité est en réalité un problème complexe; l'essentiel demeure que les clients/bénéficiaires soient capables de générer par l'ensemble de leurs activités une rentabilité suffisante pour couvrir le coût global de leur endettement.

Si l'on se penche sur les effets du couplage microcrédit-micro-assurance santé, on peut être tenté de faire un parallélisme avec ce qu'on a connu en microfinance durant plus de vingt ans en matière d'association entre crédit et formation. Les systèmes postulaient que pour être vraiment efficaces (socialement), les crédits devaient s'accompagner de formation; le coût de celle-ci venait dès lors s'ajouter automatiquement au crédit, rendant celui-ci plus cher pour tous, y compris les individus qui ne souhaitaient pas suivre cette formation (ou pour qui les formations dispensées n'étaient pas adéquates). Dans certains cas, la viabilité même de certains programmes de microfinance fut entamée. Par ailleurs, très souvent les institutions ayant ce profil trouvaient normal d'utiliser les fonds générés par les volets microfinance pour subventionner leurs autres activités (formation, aide à la commercialisation...), ces dernières ne parvenant pas à couvrir leurs coûts. Si ce choix pouvait 
sembler acceptable lorsque les services annexes constituaient une véritable valeur ajoutée pour les clients-bénéficiaires, il faut reconnaître qu'il fut contesté dans de nombreux cas, tant par les clients (optant pour des systèmes financiers sans services annexes lorsqu'ils ont eu le choix) que par les gestionnaires des systèmes de microfinance (pour qui la "confiscation" des excédents qu'ils généraient venait réduire la capacité d'auto-financement et de développement des systèmes d'épargne-crédit dans un contexte où la demande aurait pourtant justifié des politiques d'expansion autofinancées). Il s'agit donc d'analyser si le couplage n'induit pas des coûts implicites liés au crédit trop élevés, entraînant l'exclusion de certains bénéficiaires potentiels qui auraient accédé au microcrédit s'il n'y avait pas eu couplage.

\subsection{Effets du couplage sur le plan de la micro-assurance santé}

Pour la seconde famille d'hypothèses, on estime que grâce à l'accès à des services de microfinance, les personnes peuvent plus aisément accéder aux systèmes de micro-assurance santé. En effet, l'accès à la microfinance permettrait aux bénéficiaires de gérer (lisser) leurs problèmes de liquidités, et ainsi de mieux assumer leurs obligations (cotisations) dans les systèmes d'assurance santé. D'une part, il est incontestable que la microfinance peut être un outil qui facilite le paiement de la cotisation, puisqu'on peut imaginer que le coût de celle-ci soit payé en une fois pour être ensuite intégré dans le coût total du crédit. D'autre part, dans la mesure où l'accès au microcrédit faciliterait le développement d'activités génératrices de revenus, l'individu pourrait posséder plus de revenus pour faire face au paiement des cotisations (Fonteneau, 2004). Un second type d'argument justifie l'impact positif du couplage sur le plan de la protection sociale. En associant les services de microfinance et de microassurance santé, on peut faire l'hypothèse qu'on attire une population dans une démarche d'assurance santé qu'elle n'aurait pas faite par ailleurs, en particulier, les travailleurs du secteur informel. En effet, si l'emprunt est une transaction socio-économique bien ancrée dans les pratiques traditionnelles, la démarche d'assurance étant associée par définition à la probabilité d'un sinistre, est vécue négativement. Le fait de s'assurer pourrait favoriser l'apparition du sinistre. Traditionnellement, la logique de l'assurance est associée à l'existence des groupes sociaux; une fois l'occurrence du sinistre, le sinistré fonde son espoir sur la solidarité financière du groupe social auquel il appartient pour couvrir les frais liés au sinistre. En outre, la logique de l'assurance nécessite la constitution de fonds dans lesquels on viendra puiser en cas de sinistre. Ces fonds sont parfois vus comme des "fonds qui dorment", alors que dans les milieux populaires africains, on conçoit avant tout l'argent comme devant circuler.

Enfin, il est parfois ardu pour les assurés de comprendre qu'ils ont bénéficié d'un service (la couverture proprement dite), même si ils ne sont pas tombés malade. Dans ce cas, il faut souligner l'importance de travailler sur la perception qu'ont les usagers potentiels des bénéfices liés à la micro-assurance santé. 
Comment les personnes appréhendent-elles le risque maladie et sa couverture? Peut-on dire que les perceptions culturelles du crédit et de l'assurance santé sont identiques ? De ce point de vue, on peut, par exemple, faire l'hypothèse que l'articulation entre micro-assurance santé et mutuelle de santé peut induire un effet positif sur la dynamique de participation et d'appropriation des membres.

\subsection{Effets du couplage sur le plan du renforcement institutionnel}

Pour le troisième et dernier groupe d'hypothèses, grouper ces services renforce l'institution sur différents plans : les économies de coûts pour l'institution, qui peuvent se répercuter sur le prix des services, et le renforcement de la mission. D’abord, il est supposé qu'on peut bénéficier d'effets d'échelle et de champ susceptibles d'améliorer la structure de coûts et la qualité des services proposés par les systèmes de microfinance et de micro-assurance santé. D’un point de vue théorique, une telle association devrait, pour se justifier, apporter un plus au client/bénéficiaire par rapport à la fourniture des services de manière séparée et ce à un coût identique ou inférieur à la somme des coûts supportés dans le cas de services dissociés. Pour améliorer la structure de coûts, il faudrait que l'association de systèmes de microfinance et d'assurance santé engendre des synergies permettant des économies d'échelle ou de champ. Sur la base de ce que nous avons présenté dans la première partie de cet article, seuls deux domaines nous semblent susceptibles d'y participer: les coûts administratifs directs (rentabiliser un même bureau en groupant les services) et les frais de marketing (en tentant de coupler les clientèles comme ont tenté de le faire les institutions de bancassurance dans certains pays industrialisés). Reste que même si de telles économies sont possibles, il faut être conscient des risques qu'il y a à fournir ces services de manière groupée. Nous voudrions en souligner quatre.

Premièrement, la gestion de la liquidité. Déjà mentionnée, elle est un élémentclé des deux types de services. Mais les contraintes imposées aux deux services ne sont pas identiques. Il ne faudrait donc pas que pour assurer la liquidité de l'un, on mette l'autre en péril.

Deuxièmement, la gestion des actifs. En microfinance, la gestion d'actifs/passifs se fait selon les normes bancaires traditionnelles des accords de Bâle (parfois légèrement adaptées sur des points comme le Capital Adequacy Ratio ou la rapidité de constitution de provisions pour arriérés et impayés). Ce type de normes permet qu'une partie des fonds collectés au travers des programmes d'épargne soit canalisée vers des microcrédits. Dans la micro-assurance santé, par contre, les avoirs collectés doivent être gérés avec une très grande prudence pour s'assurer que les fonds seront toujours disponibles en cas de nécessité. En associant les deux, on peut craindre qu'au fil du temps, une partie (l'ensemble ?) des primes collectées en assurances santé soient perçues par les systèmes de microfinance comme une source de financement bon marché. Autre risque, une institution combinant les deux services pourrait vouloir utiliser l'épargne 
collectée pour couvrir les indemnisations liées aux contrats d'assurance santé lorsque les primes ne permettent pas d'équilibrer le système ; elle mettrait ainsi en péril les avoirs de ses épargnants. Dans les deux cas, la gestion actifs/passifs n'en deviendrait que moins transparente et plus risquée. Enfin, à moins de disposer d'une comptabilité analytique précise, on peut craindre qu'il soit difficile de répartir les coûts de manière réellement représentative de la réalité.

Troisièmement, il faut se méfier des associations faites par les clients. Ainsi, s'ils constatent que "microfinance" et "micro-assurance santé" ne font qu'un du point de vue institutionnel, on peut craindre que dans certains cas problématiques les individus qui se verraient refuser telle ou telle prestation en soins de santé décident de ne pas rembourser leur prêt (pour se faire justice) ou, à l'inverse, que les individus n'ayant pas obtenu les conditions financières souhaitées en termes de crédit se retirent du système de micro-assurance santé. Probablement minoritaires, ces cas peuvent exister.

Enfin, nous pouvons interroger les effets du couplage sur le plan de la mission des organisations. Il est fondamental de souligner que la dimension de l'intérêt général de l'assurance santé est plus marquée que dans la microfinance; toute personne devant avoir accès au nom de l'équité et de l'efficacité à une couverture soins de santé ; ce qui n'est pas nécessairement le cas pour le microcrédit. Ceci implique que les missions d'une institution de microcrédit ne sont pas entièrement compatibles avec celles des organisations de micro-assurance santé. Si la question des indigents peut se poser de manière centrale pour la micro-assurance santé, c'est-à-dire l'inclusion de personnes n'ayant pas les moyens financiers de payer les primes d'assurance, elle peut s'avérer moins pertinente pour une institution de microfinance (IMF). Se pose dès lors la question de la compatibilité d'objectifs entre ces types d'organisations.

\section{DE LA QUESTION DE LA PERTINENCE DU COUPLAGE À CELLE DE SA FORME}

Outre la pertinence de l'articulation entre microfinance et micro-assurance santé, il nous semble important de s'interroger sur les formes que pourraient prendre ces articulations. De manière théorique, nous pourrions envisager quatre cas que nous présentons ici (du plus intégré au moins intégré).

La fusion pure et simple.

Structure unique dispensant à la fois des services de microfinance et de microassurance santé. C'est une solution qui semble a priori à contre-courant de ce qui se fait depuis plusieurs années lorsqu'on veut développer une articulation entre microfinance et d'autres services non financiers.

La création de groupes de type "bolding" (pas forcément par actions).

Forme qui tenterait de tirer avantages des synergies éventuellement identifiées mais en maintenant les entités relativement distinctes pour éviter les problèmes de gestion envisagés précédemment. C'est la structure généralement adoptée 
par les organisations de microfinance qui veulent maintenir un volet "formation".

Le partenariat entre entités totalement distinctes.

Il peut s'agir, par exemple, de conventions pour partager des locaux, des fichiers clients, etc. L'idée développée ici est que les deux types d'organisation ne sont pas concurrentes (puisqu'elles agissent sur des marchés différents), mais qu'en s'associant elles peuvent être plus efficaces, chacune dans son métier.

Le développement de produits conjoints.

Partant d'une logique de "co-branding", pour utiliser un terme issu du marketing, il s'agirait de profiter des images des deux partenaires pour mettre sur le marché un produit qui tout en correspondant à un besoin des clients/bénéficiaires amènerait de nouveaux clients (ou fidéliserait les anciens) aux organisations de microfinance et aux systèmes d'assurance santé. On peut penser, ainsi, à un prêt-santé qui aurait pour objectif de couvrir (mais cette fois-là tout à fait explicitement) le coût de la cotisation au système de micro-assurance santé.

De manière complémentaire, il faut s'interroger sur la structure des articulations en fonction des organisations à l'origine du montage. En effet, on peut penser que la culture d'entreprise, les pratiques de gestion et les choix stratégiques seront dans une certaine mesure influencés par cette origine: institution de microfinance, mutuelle de santé, voire structure d'appui qui se confond parfois avec l'initiateur (Fonteneau, 2004). Étudier l'ensemble des mécanismes mis en place suppose de tenir compte de ces acteurs.

\subsection{La diversité des formes de couplage observées}

Le Bénin et le Burkina Faso forment un bloc important de l'UEMOA, dans le champ de la microfinance et de la micro-assurance santé. Du point de vue de la microfinance, ces deux pays totalisent $469 \mathrm{IMF}$ aux formes variées qui servent $36,5 \%$ de part de marché global (en nombre de clients) avec 45\% d'encours de crédits de l'UEMOA (BCEAO, 2005). Ces IMF évoluent dans un contexte de forte concurrence, amenant certaines d'entre elles qui réalisent de faibles performances suivies d'une dégradation de leur portefeuille, à engager des mutations parfois profondes. Du point de vue de la micro-assurance santé, le Bénin et le Burkina comptent respectivement 43 et 136 mutuelles fonctionnelles qui sont, comme dans toute l'Afrique de l'Ouest, des systèmes relativement jeunes de nature non lucrative, fonctionnant selon les principes mutualistes (Fonteneau, 2004). En général, ces mutuelles sont de faible taille, avec une moyenne de 1009 bénéficiaires par mutuelle au Bénin, contre 448 au Burkina. Ce constat s'explique, entre autres, par "la faible capacité contributive des membres, l'irrégularité dans le paiement des cotisations, l'analphabétisme, la qualité des soins offerts par les structures sanitaires accessibles et/ou abordables pour les membres, etc." (Ibid., ix).

En vue d'analyser les diverses formes de couplage, cinq expériences ont été visitées, à savoir quatre au Bénin et une au Burkina Faso.

Le premier modèle de couplage est impulsé par des mutuelles de santé. Ainsi, 
chez PROMUSAF4, au Bénin, le couplage consiste à introduire le microcrédit dans les mutuelles de santé avec l'appui technique d'un opérateur agréé de microfinance (la Mutuelle pour le Développement à la Base, MDB), en vue de renforcer la capacité des membres à assurer le paiement régulier des cotisations. Le but recherché est d'améliorer l'accès des populations aux soins de santé de qualité à moindre coût. PROMUSAF assure l'encadrement de 17 mutuelles de santé et reçoit, à ce titre, des subventions de la coopération internationale, qui viennent renforcer le bénévolat des membres des organes de gestion des mutuelles à la base. Toutefois, malgré ces appuis, la viabilité des mutuelles de santé de PROMUSAF reste précaire, notamment compte tenu des difficultés des membres à payer leurs cotisations. En témoigne la faiblesse du taux de recouvrement des cotisations ${ }^{5}$ qui avoisine 58\% en 2004 pour l'ensemble des mutuelles. Parmi les causes de ce constat, on cite souvent la précarité 6 des revenus des activités agricoles, ainsi que la mauvaise qualité des services offerts par les centres de santé conventionnés (mauvais accueil surtout); sans oublier que le mode de collecte des cotisations induit des coûts de déplacement non couverts pour les bénévoles.

Face à ce constat de la difficulté de recouvrement des cotisations, le produit microfinance a été introduit. Grâce à la mise à disposition d'un fonds issu de la coopération internationale, des petits prêts productifs $(37 €)$ sont proposés aux mutualistes au taux d'intérêt annuel de $10 \%$. Ce taux permet de rétribuer le fonds de crédit $(2 \%)$ et de rémunérer le suivi technique de MDB (8\%). L'obtention du microcrédit est subordonnée au paiement régulier des cotisations à la mutuelle.

Le deuxième modèle de couplage est, quant à lui, impulsé par une institution de microfinance. Ainsi, AssEF7 (Bénin) et MUFEDEB ${ }^{8}$ (Burkina-faso), toutes les deux IMF de forme mutualiste, visent à construire un système financier pérenne en faveur des femmes pauvres, exclues des banques classiques. En 2003, AssEF (26 000 membres) a totalisé 1,3 milliards F CFA d'encours de crédit, le crédit moyen étant de $64 €$. MUFEDEB (17 000 membres) est une initiative des femmes licenciées de l'ex-Banque nationale de développement et a totalisé 141 millions F CFA d'encours de crédit en 2003, le crédit moyen étant de $38,1 €$. L'une de ses récentes innovations a été d'élargir son portefeuille d'activité à la tontine traditionnelle, plus précisément à la tontine itinérante.

\footnotetext{
${ }^{4}$ Le PROMUSAF (Programme d'appui aux Mutuelles de Santé en Afrique de l'Ouest) est un volet du programme d'économie sociale de l'ONG belge "Solidarité Mondiale".

${ }^{5}$ Rapport entre le montant des cotisations effectivement perçues et celui des cotisations émises (dues à date).

${ }^{6}$ Les paiements des cotisations ayant lieu en fin de cycle de production, certains membres mutualistes ont suggéré une annualisation des cotisations, pour parer aux retards enregistrés sur les paiements mensuels.

${ }^{7}$ AssEF (Association d'Entraide aux Femmes) est une IMF initiée au Bénin par l'organisation néerlandaise de développement.

${ }^{8}$ MUFEDEB : Mutualité Femmes et Développement du Burkina Faso.
} 
Le but recherché du couplage est de mettre à la disposition des membres un produit assurance santé qui puisse atténuer leur vulnérabilité et ainsi accroître leurs capacités à honorer les délais de remboursement des crédits. En effet, la maîtrise du taux d'impayés constitue le principal défi de viabilité auquel les IMF doivent faire face dans la gestion de leur croissance. Chez AssEF, par exemple, la dégradation du portefeuille de crédit a entraîné la fermeture de certaines caisses (Louis dit Guérin O., 2006). Il s'en est suivi une crise qui a conduit l'institution à adopter un plan de redressement en cours de mise en œuvre. Chez AssEF, la micro-assurance santé est intégrée dans l'organigramme de l'IMF et s'appuie donc sur le personnel et les structures du réseau de microfinance pour la collecte des cotisations et la diffusion des informations. Chez MUFEDEB, par contre, la micro-assurance santé jouit du statut juridique indépendant (association loi 1901). C'est donc une structure indépendante affiliée au réseau d'appui aux mutuelles de santé (RAMS/BF) dont elle reçoit des appuis. Cependant, la gestion des mutuelles de santé est déléguée à un employé détaché de l'IMF à cet effet, les salaires étant entièrement couverts par l'IMF, en plus des temps que le staff de l'IMF consacre aux réunions sur les mutuelles de santé sans facturation. Ici, l'obtention du crédit est subordonnée au paiement régulier des cotisations à la mutuelle de santé, tandis que chez AssEF, cette contrainte, qui n'était qu'implicite, a rapidement été levée. Par ailleurs, on observe, chez MUFEDEB, l'existence d'un système d'épargne santé, en plus du mécanisme d'assurance, spécifique à Ouagadougou, fixant les cotisations mensuelles à $2000 \mathrm{~F} \mathrm{CFA}$, dont 75\% sont réservés à la prise en charge des épargnants.

La dernière forme de couplage est observée dans deux initiatives au Bénin, dont l'une, dans la région du Mono, soutenue par Louvain Développement (LD) en partenariat avec l'ONG locale GROPERE et l'autre, plus étendue, promue par le CBDIBA9 . Elle se traduit par la création de deux systèmes parallèles, l'un de microfinance et l'autre de micro-assurance santé, en vue de générer des synergies positives au profit des couches vulnérables. Toutefois, si dans le premier cas (LD), la création de la caisse d'autopromotion d'épargne et de crédit et de la mutuelle de santé est quasi simultanée, dans le second cas (CBDIBA), le système de microfinance est largement plus ancien par rapport à la micro-assurance santé. De plus, ici, les deux volets sont indépendants, tant du point de vue de leurs statuts juridiques que de leurs organes de décision. Par ailleurs, l'assurance santé est spécifique, dans la mesure où la couverture des soins est limitée au triple du montant des cotisations, le surplus étant accordé sous forme de crédit remboursable par le patient membre.

Dans le cas LD, l'obtention du crédit à la microfinance est subordonnée au paiement régulier des cotisations à la mutuelle de santé ; toutefois, le mutualiste peut obtenir du crédit dès lors que les arriérés des cotisations peuvent être

\footnotetext{
${ }^{9}$ CBDIBA (Centre Béninois pour le Développement des Initiatives à la Base) est une ONG visant à promouvoir le développement intégré des populations à la base du Bénin. Une de ses initiatives est l'organisation d'un système de crédit-santé.
} 
déduits du montant de crédit sollicité. Par contre, au CBDIBA, cette obligation n'est faite que pour le "Crédit Adhésion Progressivee" spécifiquement conçu pour les personnes n'ayant aucune garantie matérielle. L'accès aux autres produits des caisses d'épargne et de crédit n'est pas subordonné à cette obligation. Cela s'explique par le fait que ces caisses, bien plus anciennes, sont organisées en réseau, alors que certaines seulement sont en partenariat avec le réseau des associations de micro-assurance santé communautaire, ces dernières n'existant pas encore dans toutes les localités disposant des premières.

\subsection{Les effets du couplage}

Nous avons observé dans les diverses formes de couplage un alignement sur les objectifs de la structure faittière: lorsqu'une mutuelle de santé développe une activité de microcrédit, elle veillera à l'accès à une population très vulnérable, cible de la mutuelle de santé (par exemple chez PROMUSAF); lorsqu'une coopérative d'épargne et de crédit offre un produit assurance santé, son but premier est d'offrir un produit supplémentaire à ses membres et d'améliorer la qualité de son portefeuille de crédit.

Les différentes expériences montrent comment le microcrédit constitue un mécanisme incitatif important pour fidéliser les membres de la mutuelle de santé. En effet, il apparaît que, dans plusieurs cas, le couplage attire des personnes vers la micro-assurance santé qui n'auraient pas contracté une assurance santé par ailleurs. On peut, cependant, se demander si la participation à l'assurance santé est toujours valorisée en tant que telle ou si elle n'est, en fait, qu'un coût de transaction caché rendu nécessaire lorsque l'accès au crédit est soumis à l'obligation d'adhérer à l'assurance santé. Il apparaît, d'ailleurs dans certains cas, que le coût implicite du crédit est trop élevé pour les microentrepreneurs les plus fragiles qui renoncent alors au microcrédit. Cependant, cette obligation peut être aussi l'occasion de convaincre des personnes du bienfondé d'une assurance santé. C'est ainsi que les personnes qui tomberaient malades lors de cette période où elles sont obligées d'être assurées pourraient renouveler leur adhésion, même après le remboursement du crédit. La spécificité de l'assurance santé de CBDIBA, qui s'inspire des systèmes d'épargne santé, a l'avantage d'être plus proche des pratiques endogènes en termes d'épargne et de crédit, mais ce dispositif produit des effets limités en terme de partage des risques et de la consolidation des principes mutualistes.

Nous n'avons pas pu rassembler les données nécessaires pour vérifier l'hypothèse, d'une part, que l'accès au crédit renforçant la capacité productive des micro-entrepreneurs, facilite leur adhésion et améliore les paiements des cotisations à la mutuelle de santé et, d'autre part, que l'adhésion à la mutuelle de santé réduit les risques de défaut par rapport au remboursement des microcrédits. Si certaines économies d'échelle via l'articulation ont pu être observées en termes de mise en commun d'information (fichier clients) ou d'équipement, des questions se posent par rapport à la durabilité des systèmes mis en place. D’une part, il est clair qu'un système de microcrédit à faible taux, 
tel que pratiqué chez PROMUSAF, a de faibles chances d'être durable, et qu'en le développant, on risque d'entraver l'émergence d'autres organisations de microfinance susceptibles d'être pérennes. D’autre part, malgré des efforts de répartition des coûts (notamment lorsque les structures sont distinctes juridiquement) ou de séparation des comptabilités, on observe que le financement des mutuelles de santé absorbe, dans de nombreux cas, une part des ressources issues de la microfinance.

\section{CONCLUSION}

Pour de nombreuses populations de pays en développement, avoir accès à des soins de santé décents et disposer de services financiers répondant à leurs besoins constituent de réelles priorités. On peut donc parfaitement comprendre que les acteurs intervenant dans ces domaines envisagent de combiner ces types d'appui. Reste que ces associations peuvent prendre de nombreuses formes et soulèvent de multiples questions qui mériteraient d'être approfondies. À ce stade, nous en retiendrons quatre.

Quelles sont les conditions nécessaires pour que l'articulation entre une institution de microfinance et une mutuelle de santé (ou une autre structure d'assurance santé) apporte une véritable amélioration pour les bénéficiaires de ces services?

Comment peut-on s'assurer qu'un modèle d'articulation participe du renforcement des deux volets considérés et non pas d'une logique visant à privilégier l'un au détriment du développement de l'autre?

En présence d'un nombre de clients/bénéficiaires et/ou adhérents limité, quels sont les critères qui devraient permettre de choisir entre une stratégie de mutualisation réalisée directement, au travers de l'établissement d'une mutuelle de santé, et une stratégie visant à atteindre cette même mutualisation, mais à terme au travers de la mise en œuvre préalable d'un mécanisme d'épargne-santé basé sur une logique de pré-paiement?

Enfin, pour les organisations ayant fait le choix de l'articulation entre microfinance et assurance santé, quelle structure faut-il privilégier pour permettre une croissance saine et contrôlée susceptible de répondre aux besoins exprimés?

\section{BIBLIOGRAPHIE}

AMEGBEDJI U. S. (2004) Inventaire des systèmes d'assurance maladie en Afrique. Rapport du Bénin, Document de la Concertation entre les acteurs de développement des mutuelles de santé en Afrique, 42 pages.

AssEF (2006) Plan de redressement de l'association d'entraide des femmes, septembre, 58 pages.

ATIM C. (2000) Contribution des mutuelles de santé au financement, à la fourniture et à l'accès aux soins de santé : synthèse de travaux de recherche menés dans neuf pays d'Afrique de l'ouest et $d u$ centre, USAID, BIT, WSM, ANMC Belgique, Bruxelles, mars, 78 pages. 
BANQUE MONDIALE (1998) Stratégie de la Banque mondiale pour le développement de la microfinance et des services financiers aux PME et en milieu rural en Afrique subsabarienne, Rapport, $\mathrm{n}^{\circ}$ 21202, Washington DC., août.

BCEAO (2005) Notes d'information statistiques, Les principaux indicateurs économiques et financiers du Bénin, avril, www.bceao.int.

BOTZUNG M. (1996) Dispositifs d'appui et financement de la petite entreprise, Tiers Monde, Tome XXXVII, 145, 135-152.

BROWN W. \& CHURCHILL C. F. (2004) Quelles leçons tirer des premières expériences de micro-assurance? AD A Dialogue, $\mathrm{n}^{\circ}$ 33, Luxembourg, pp. 55-78.

CELLULE MICROFINANCE (2003) Bilan et perspectives à court et moyen termes de la microfinance au Bénin. Évaluation et impacts de la microfinance sur les bénéficiaires de microcrédits, PADSP - BEI, Cotonou.

CES, ESP, ISPEC, IRSP (2004) La formation et la recherche au service du développement de la micro-assurance santé au Bénin, Actes de colloques, décembre, Ouidah, 53 pages.

CHURCHILL C. F. (2004) La microfinance est-elle une priorité pour les pauvres ? Comprendre la demande de services financiers de gestion des risques, $A D A$ Dialogue, $\mathrm{n}^{\circ} 33$, juin, Luxembourg, 11-30.

CHURCHILL C. F. (2005) Les institutions de microfinance doivent-elles proposer des services d'assurance? Techniques Financières et Développement, Epargne Sans Frontière, $\mathrm{n}^{\circ}$ 78, mars, 99-115.

CHURCHILL C. F., LIBER D., Mc CORD M. J., ROTH J. (2004) L'assurance et les institutions de microfinance : guide technique pour le développement et la prestation de services en micro-assurance, Genève, ILO \& ADA (pour la traduction française), 250 pages.

DROR M. D. \& PREKER S. A. (2002) Social Reinsurance: A New Approach to Sustainable Community Health Financing, Geneva \& Washington D.C., ILO \& The World Bank.

FONTENEAU B., avec la collaboration de Coumba Gueye, Djakaridja Hema Ouattara et Aboubakar Koto-Yérima, (2004) Les défis des systèmes de micro-assurance santé en Afrique de l'Ouest : cadre politique, environnement institutionnel, fonctionnement et viabilité, Kuleuven-HIVA-VLIR-DGCD, mars, 360 pages.

GROUPE DE TRAVAIL SUR LA MICRO-ASSURANCE (2004) Lignes directrices préliminaires en micro-assurance, destinées aux bailleurs de fonds, $A D A$ Dialogue, $\mathrm{n}^{\circ} 33$, juin, Luxembourg, 79-94.

JAFFRE Y., OLIVIER DE SARDAN J-P. (2003) Une médecine inhospitalière : les difficiles relations entre soignants et soignés dans cinq capitales de l'Afrique de l'Ouest, Paris, APADKarthala, 457 pages.

LABIE M. (2004) Microfinance : un état des lieux, Mondes en Développement, Tome 32, $\mathrm{n}^{\circ}$ 126, 9-23.

LABIE M., NGONGANG I., NYSSENS M., WELE P. (2006) Analyser l'articulation entre microfinance et micro-assurance santé : réflexions à partir de trois cas béninois, Document d'économie et de gestion, Working Paper 2006/2, Centre de Recherche Warocqué, 41 pages.

LAI M. (2000) Conférence virtuelle sur la micro-assurance, $A D A$ Dialogue, $\mathrm{n}^{\circ} 24$, décembre, Luxembourg, 29-40.

LOUVAIN DEVELOPPEMENT, PROMUSAF (2002) Développement des mutuelles de santé, rôles et places des acteurs dans les relations avec les prestataires de soin, rapport général, 62 pages. 
LOUIT dit GUERIN O. (2006) Association d'entraide des femmes, Bénin, CGAP Working Group on Microinsurance, Good and Bad Practices, Case Study n ${ }^{\circ} 22$.

MILLER M. \& NORTHRIP Z. (2000) L'assurance comme produit de microfinance, Note Technique $n^{\circ}$ 4, Série Innovations en Microfinance, Bamako, 18 pages.

POURSAT C. (2004) Micro-assurance santé et microfinance : quelques enseignements du programme pilote du GRET au Cambodge, in Guérin \& Servet, Exclusion et liens financiers, Rapport 2003 du Centre Walras, Paris, Economica, 56-66. 